\title{
TESTING FOR DIFFERENCES IN SELECTIVITY DURING MASS AND BACKGROUND EXTINCTIONS USING THE RELATIONSHIP BETWEEN SIZE AND EXTINCTION INTENSITY IN TRILOBITA
}

CHURCHILL, Lisa L., Museum of Paleontology, University of Michigan, Ann Arbor, MI 48109, U.S.A.

The existence of separate selectivity regimes during mass and background extinctions remains a controversial topic in paleontology. While most studies recognize that there is a continuous distribution between the two (in terms of extinction intensity), the question still remains whether the evolutionary processes operating during mass extinctions are simply an intensification of those seen during background extinctions, or whether there exist two separate selectivity regimes, one active during periods of great extinction and the other during lesser extinctions. The possible existence of two selection regimes implies that a biological threshold may also exist, such that once crossed, evolution no longer behaves in the same manner.

In an effort to address this subject, the relationship between changes in size and extinction intensity in Trilobita was analyzed. Specifically, changes in variances and means between the preextinction and post-extinction generic populations were plotted against the corresponding extinction intensities to determine if there was a change in the direction or type of selectivity with an increasing generic cull. This approach differs from previous studies since the time element was eliminated from the plots. The pattern seen in the fossil record was also compared to those produced by computer simulations in an effort to better identify the type of selection observed.

No distinction could be made between the patterns of selectivity seen in the fossil record and those produced by stochastic computer simulations. This implies that selectivity alone cannot be used to distinguish between mass and background extinctions. However, directed selection was observed in five of the eleven intervals studied, confirming that not all extinctions can be adequately explained under the random model (significant changes are indicated by an asterisks in the figures below). In each of these cases, selection was directed against the larger morphotypes, as evidenced by a significant decrease in mean size. A strong correlation between significant changes in mean and generic extinction intensity was also discovered, revealing that as the extinction intensity increased, so did the amount of negative change in mean. This suggests that, on average, smaller forms were better able to withstand extinction pressures than their larger counterparts and that this advantage increased with greater extinction intensities. The fact that no significant changes in variance were observed implies that the overall range of generic sizes remained the same, regardless of the level of extinction or the direction of selectivity.

Change in Mean Size vs. Extinction Intensity

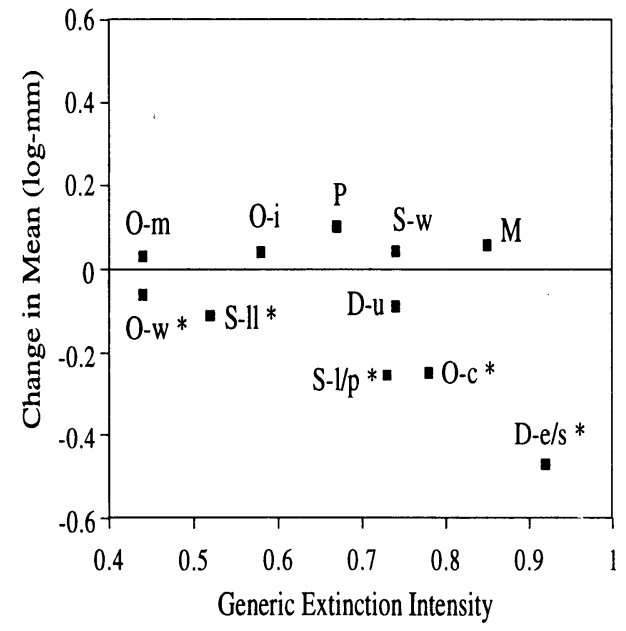

Change in Variance of Sizes vs. Extinction Intensity

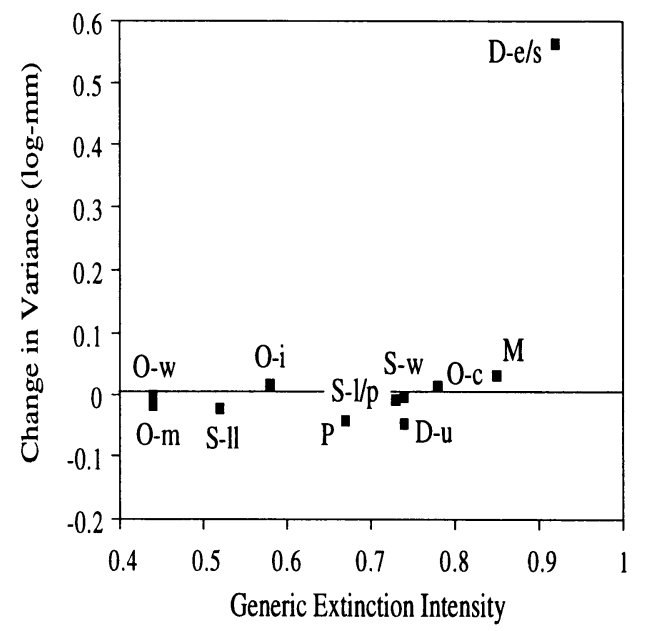

